\title{
Ionizing Radiation Stimulates Expression of Pro-Osteoclastogenic Genes in Marrow and Skeletal Tissue
}

\author{
Joshua S. Alwood, * Mohammad Shahnazari, ${ }^{*}$ Betsabel Chicana, A.S. Schreurs, \\ Akhilesh Kumar, Alana Bartolini, Yasaman Shirazi-Fard, and Ruth K. Globus
}

Exposure to ionizing radiation can cause rapid mineral loss and increase bone-resorbing osteoclasts within metabolically active, cancellous bone tissue leading to structural deficits. To better understand mechanisms involved in rapid, radiation-induced bone loss, we determined the influence of total body irradiation on expression of select cytokines known both to stimulate osteoclastogenesis and contribute to inflammatory bone disease. Adult (16 week), male C57BL/6J mice were exposed to either $2 \mathrm{~Gy}$ gamma rays $\left({ }^{137} \mathrm{Cs}, 0.8 \mathrm{~Gy} / \mathrm{min}\right)$ or heavy ions $\left({ }^{56} \mathrm{Fe}, 600 \mathrm{MeV}, 0.50-1.1 \mathrm{~Gy} / \mathrm{min}\right)$; this dose corresponds to either a single fraction of radiotherapy (typical total dose is $\geq 10 \mathrm{~Gy}$ ) or accumulates over long-duration interplanetary missions. Serum, marrow, and mineralized tissue were harvested $4 \mathrm{~h}-7$ days later. Gamma irradiation caused a prompt (2.6-fold within $4 \mathrm{~h}$ ) and persistent (peaking at 4.1-fold within 1 day) rise in the expression of the obligate osteoclastogenic cytokine, receptor activator of nuclear factor kappa-B ligand (Rankl), within marrow cells over controls. Similarly, Rankl expression peaked in marrow cells within 3 days of iron exposure (9.2-fold). Changes in Rankl expression induced by gamma irradiation preceded and overlapped with a rise in expression of other pro-osteoclastic cytokines in marrow (eg, monocyte chemotactic protein-1 increased by 11.9 -fold, and tumor necrosis factoralpha increased by 1.7-fold over controls). The ratio, Rankl/Opg, in marrow increased by 1.8-fold, a net proresorption balance. In the marrow, expression of the antioxidant transcription factor, $N f e 2 l 2$, strongly correlated with expression levels of Nfatcl, Csfl, Tnf, and Rankl. Radiation exposure increased a serum marker of bone resorption (tartrate-resistant acid phosphatase) and led to cancellous bone loss (16\% decrement after 1 week). We conclude that total body irradiation (gamma or heavy-ion) caused temporal elevations in the concentrations of specific genes expressed within marrow and mineralized tissue related to bone resorption, including select cytokines that lead to osteoclastogenesis and elevated resorption; this is likely to account for rapid and progressive deterioration of cancellous microarchitecture following exposure to ionizing radiation.

\section{Introduction}

$\mathrm{D}$ URING A SPACEFLIGHT BEYOND the Earth's protective magnetosphere, astronauts are exposed to a complex mixture of ionizing radiation (Durante and Cucinotta 2011), including low-linear energy transfer (LET) gamma rays and protons, as well as more damaging high-LET radiation. Exposure to space radiation is characterized by relatively low doses $(\leq 2 \mathrm{~Gy})$ of ion species due to solar particle events (Shurshakov and others 1999; Parsons and Townsend 2000) or galactic cosmic rays (Zeitlin and others 2013; Hassler and others 2014). Simulated space radiation at these doses causes a rapid net decline in cancellous bone volume to total volume as well as a decrement relative to age-matched controls (Kondo and others 2009; Willey and others 2010). The bone loss is associated with increased osteoclast numbers and resorbing surfaces of osteoclasts lining trabeculae (Hamilton and others 2006; Alwood and others 2010; Kondo and others 2010; Willey and others 2010; Yumoto and others 2010; Lloyd and others 2012). Doses in the range of

Bone and Signaling Laboratory, Space Biosciences Division, NASA Ames Research Center, Moffett Field, California.

*Authors share first authorship.

(C) Joshua S. Alwood et al. 2015; Published by Mary Ann Liebert, Inc. This Open Access article is distributed under the terms of the Creative Commons License (http://creativecommons.org/licenses/by/4.0), which permits unrestricted use, distribution, and reproduction in any medium, provided the original work is properly credited. 
1-2 Gy also are relevant to radiotherapy; total therapeutic doses can vary, but total body doses of 10-15 Gy typically are fractionated into single doses of $\sim 2 \mathrm{~Gy}$, which ultimately can lead to increased fracture incidence (Baxter and others 2005). Exposure to radiation, particularly high-LET particles, has the potential to exacerbate the deleterious effects of musculoskeletal disuse, which occurs during prolonged bed rest or spaceflight (LeBlanc and others 2000; Lang and others 2004, 2006; Keyak and others 2009; Alwood and others 2010; Yumoto and others 2010).

Previous work shows that radiation exposure elicits a transient $(<2$ weeks) pro-resorptive state in cancellous tissue, resulting in net bone loss compared with basal (time of radiation) and age-matched controls (Kondo and others 2010; Willey and others 2010; Alwood and others 2012). Bone-resorbing osteoclasts are thought to cause the rapid (Kondo and others 2009; Willey and others 2010; Turner and others 2013) cancellous strut losses following simulated space irradiation, leading to loss of microarchitectural integrity (Alwood and others 2010). Radiation exposure transiently and markedly increases the numbers of osteoclasts and the extent of cancellous surfaces covered by osteoclasts. However, the role that receptor activator of nuclear factor kappa-B ligand (Rankl), the principal osteoclastogenic cytokine, plays in concert with other pro-osteoclastic inflammatory cytokines (Kim and others 2006; Takayanagi 2007; Boyce and Xing 2008) and oxidative stress is not fully understood with respect to the cancellous bone loss following radiation exposure (Kondo and others 2009).

Total body irradiation ( $\leq 2 \mathrm{~Gy}$ ) causes time-dependent changes in the bone formation by osteoblasts, although these changes do not account for the early and rapid decrement in bone mass observed following exposure to total body irradiation within this dose range. Within 3 days of exposure to radiation, the net bone formation rate to bone surface (BFR/ $\mathrm{BS}$ ) is unaffected (Kondo and others 2010). Although radiation does affect osteoblast function within the first week of exposure, as indicated by an observed increase in mineral apposition rate (MAR), a concomitant reduction in the mineralizing surface yields no net change in the bone formation rate (Kondo and others 2010). Similarly, Willey and others (2010) also report increased MAR within the first week after exposure, but subsequently BFR/BS declines by 2 and 3 weeks after exposure to 2 Gy X-ray, while Turner and others reported transient increased bone formation even after very high doses of radiation $(6 \mathrm{~Gy})$. Therefore, radiation-induced changes in bone formation are more likely to contribute to later remodeling deficits rather than acute bone loss.

To better understand net bone loss in the acute response to radiation exposure, we examined the temporal expression within both marrow and mineralized tissue of Rankl and select pro-osteoclastogenic cytokines implicated in various models of inflammatory bone loss (Braun and Schett 2012) following exposure to low- or high-LET species of radiation. Furthermore, we investigated the temporal expression of a key antioxidant transcription factor (nuclear factor, erythroidderived 2,-like 2, Nfe2l2), which is responsible for mounting a rapid defense against oxidative challenges (Kobayashi and Yamamoto 2005) and is known to play a role in dampening osteoclastogenesis (Hyeon and others 2013).

We hypothesized that radiation exposure induces expression of pro-osteoclastogenic, pro-inflammatory, and antioxidant genes within both marrow and mineralized tissue compartments and increases markers of bone resorption. Furthermore, we hypothesize that these changes are likely to contribute to later cancellous bone loss. This work shows an acute and time-dependent elevation of Rankl, monocyte chemotactic protein-1 (Mcpl), and tumor necrosis factoralpha $(\operatorname{Tn} f)$ gene expression in the marrow or in skeletal compartments due to low- or high-LET irradiation. These molecular changes precede ( $<3$ days) the manifestation of bone loss (3-7 days) following iron irradiation at a dose relevant to fractionated radiotherapy or space missions.

\section{Materials and Methods}

\section{Animals}

Post-pubescent (16 weeks \pm 4 days at time of irradiation), male C57BL/6J mice (Jackson Laboratories) were individually housed and provided food (LabDiet 5001) and water ad libitum, as described elsewhere (Yumoto and others 2010). Animals were euthanized by $\mathrm{CO}_{2}$ inhalation or anesthetized with isoflurane, followed by blood draw through cardiac puncture. The Institutional Animal Care and Use Committees for NASA Ames Research Center and Brookhaven National Laboratory approved all procedures.

\section{Experiment design and radiation exposure}

Experiments were conducted to determine the temporal changes in the levels of key genes and circulating proteins related to bone resorption in the latency period before the onset of overt structural loss. To evaluate heavy-ion effects, conscious mice were exposed to high-LET iron ions $\left({ }^{56} \mathrm{Fe}\right.$, $600 \mathrm{MeV} /$ ion, $5 \mathrm{cGy}$ or $2 \mathrm{~Gy}$, at a rate of 5 or $0.50-1.10 \mathrm{~Gy} /$ min, respectively) at the NASA Space Radiation Laboratory, Brookhaven National Laboratory, or were sham irradiated as previously described ( $n=5-8 /$ group). Mice were euthanized and tissues harvested at baseline (ie, time of irradiation) or 3 or 7 days after exposure. To evaluate gamma radiation effects, conscious mice were irradiated with lowLET ${ }^{137} \mathrm{Cs}$ gamma rays [ $2 \mathrm{~Gy}, 0.80 \mathrm{~Gy} / \mathrm{min}$, as described in detail in Kondo and others (2010)] or were sham irradiated. Euthanasia and tissue harvest took place $4 \mathrm{~h}$ or 1 day $( \pm 2 \mathrm{~h})$, 3 days, or 7 days after exposure ( $n=5-7$ /group).

\section{Bone microarchitecture}

Bone volume and microarchitecture of the proximal tibial metaphysis were quantified by microcomputed tomography $(6.8 \mu \mathrm{m}$ pixel size, $3,500 \mathrm{~ms}$ integration time, $50 \mathrm{kV}$, Skyscan 1174; Bruker microCT) similar to Kondo and others (2009). Briefly, a $1.0-\mathrm{mm}$ thick region located $0.24 \mathrm{~mm}$ distal to the proximal growth plate of the tibia was selected and semiautonomously contoured to include cancellous tissue. To assess bone loss, the bone volume to total volume fraction (BV/TV, \%), trabecular thickness (Tb.Th, $\mu \mathrm{m})$, trabecular number (Tb.N, 1/mm), and trabecular separation (Tb.Sp, $\mu \mathrm{m}$ ) were calculated and reported following conventional guidelines (Bouxsein and others 2010).

\section{qRT-PCR for gene expression within marrow and skeletal tissue}

Femora and tibiae were dissected, cleaned of soft tissues, flushed of bone marrow with phosphate-buffered saline, 
and stored in RNALater (Qiagen, Inc.) at $-80^{\circ} \mathrm{C}$. Bone marrow cells were lysed and preserved with guanidinethiocyanate-containing RLT buffer (Qiagen, Inc.) with 1\% beta-mercaptoethanol at $-80^{\circ} \mathrm{C}$. RNA was extracted from homogenized bone or marrow lysates using Trizol (Ambion), QIAshredder, and an RNeasy mini kit (Qiagen, Inc.). For each tissue, RNA was treated with an RNase-free DNase set (Qiagen, Inc.) in accordance with the manufacturer's instructions. RNA quality and quantity were determined using a spectrophotometer (NanoDrop). The RNA quality was confirmed by electrophoresis using the 2100 Bioanalyzer (Agilent Technologies).

Following the manufacturer's recommendations, RNA was reversed transcribed and simultaneously used for qPCR using the GoTaq ${ }^{\circledR}$ Probe 1-Step RT-qPCR system (Promega). Portions of the following mouse gene sequences were amplified using TaqMan gene expression assays (Applied Biosystems, Inc.): receptor activator of nuclear factor kappa-B ligand (Rankl, assay ID: Mm00441906_m1), Osteoprotegerin (Opg, assay ID: Mm01205928_m1), Tumor necrosis factoralpha (Tnf, assay ID: Mm00443260_g1), monocyte chemotactic protein-1 (Mcp1, assay ID: Mm00441242_m1), interleukin-6 (Il6, assay ID Mm00446190_m1), tartrate-resistant acid phosphatase (Acp5, assay ID: Mm00475698_m1), cathepsin-K (Ctk, assay ID: Mm00484039_m1), nuclear factor of activated Tcells, cytoplasmic 1 (Nfatc1, assay ID: Mm00479445_m1), nuclear factor, erythroid-derived 2,-like 2 ( $N f e 2 l 2$, assay ID: Mm00477784_m1), and colony-stimulating factor 1 (Csf1, assay ID: Mm00432686_m1). We standardized gene expression levels to mitochondrial ribosomal protein L19 (L19, assay ID: Mm02601633_g1) to correct for differences in cellular input and RNA quality and facilitate comparison between samples. Hypoxanthine-guanine phosphoribosyltransferase (Hprt1, assay ID: Mm01545399_m1) and transmembrane protein 40 (Tmem40, assay ID: Mm00460636_m1) also were analyzed as alternate housekeeping genes. The reactions were performed in the 7300 RT-PCR system (Applied Biosystems) or SmartCycler real-time PCR system (Cepheid).

We analyzed multiple candidate housekeeping genes for normalization, including L19, Hprt1, and Tmem40. Gamma radiation exposure did not modulate levels of the gene $L 19$ at the various time points, but transiently and modestly increased gene expression of Hprtl (-0.4 cycles, 1.4-fold) and Tmem40 (-1 cycle, 2.3-fold). Following iron irradiation, L19 (as well as Hprt1) showed small increases in cycle number due to treatment (-0.4 cycles, 1.4-fold for L19). As these differences in housekeeping gene expression were small relative to those of cytokine and resorption marker levels, results reported were normalized relative to $L 19$ for both gamma and iron irradiation experiments.

\section{Serum tartrate-resistant acid phosphatase 5b}

Blood was collected from the heart at the time of euthanasia and serum was separated and stored at $-80^{\circ} \mathrm{C}$ until processed. Enzyme immunoassays were performed for measurement of tartrate-resistant acid phosphatase $5 \mathrm{~b}$ (TRACP 5b), a biomarker for osteoclast-mediated bone resorption, using a commercial kit (Immunodiagnostic Systems) according to the manufacturer's protocol.

\section{Statistics}

All data are reported as mean \pm standard deviation. To determine significant differences compared with shamirradiated controls, a 1-way analysis of variance was used followed by Dunnett's post hoc test. If data were determined to be heteroscedastic (Bartlett's test), a Welch's ANOVA test followed by a post hoc test using the Bonferroni correction were used to determine significant differences from the sham-irradiated controls. Correlation analyses were performed on expression levels of select genes from marrow and bone specimens following gamma irradiation (for the sham and 1, 3, and 7 day endpoints) using the Pearson's product-moment coefficient (r). A $P$ value $\leq 0.05$ was accepted as significant for all analyses. All analyses were performed using JMP 8.0.2 (SAS).

\section{Results}

\section{Cancellous microarchitecture following iron irradiation}

To determine the extent of bone loss over the short term, mice were irradiated with ${ }^{56} \mathrm{Fe}$ ions $(600 \mathrm{MeV})$ or were sham irradiated ( 0 Gy controls), then 7 days later bones were harvested and cancellous microarchitecture in the proximal tibia quantified ex vivo using 3D microcomputed tomography. Body weights of irradiated and control animals at the time of tissue harvest did not differ (data not shown). Compared with sham controls, irradiation with 2 Gy reduced BV/TV by $16 \%$, Tb.N by $15 \%$, and showed a trend of increased Tb.Sp by $11 \%$ $(P=0.053$, ANOVA), but did not affect trabecular thickness (Fig. 1). This is consistent with previous reports (Hamilton and others 2006; Alwood and others 2010). We included a basal group in our experiments showing BV/TV of $25.1 \% \pm$ $3.6 \%$, which was not different from the age-matched sham control group. A lower dose of iron $(5 \mathrm{cGy})$ failed to elicit changes in bone structural parameters compared with controls and is therefore below the threshold dose for causing bone loss (data not shown). These results validate the model of ionizing radiation-induced cancellous bone loss.
FIG. 1. $2 \mathrm{~Gy}$ iron irradiation caused acute bone loss in the tibial metaphysis by 7 days through removal of trabecular struts. (A) Bone volume fraction (BV/TV), (B) trabecular number (Tb.N), (C) trabecular thickness (Tb.Th), and (D) trabecular spacing (Tb.Sp). Data are mean $\pm \mathrm{SD}$, with $*$ denoting $P<0.05$ versus sham.
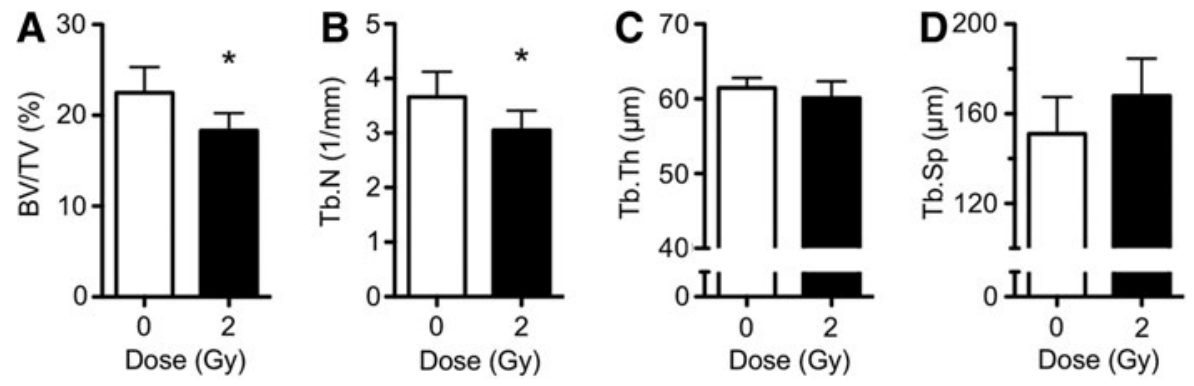


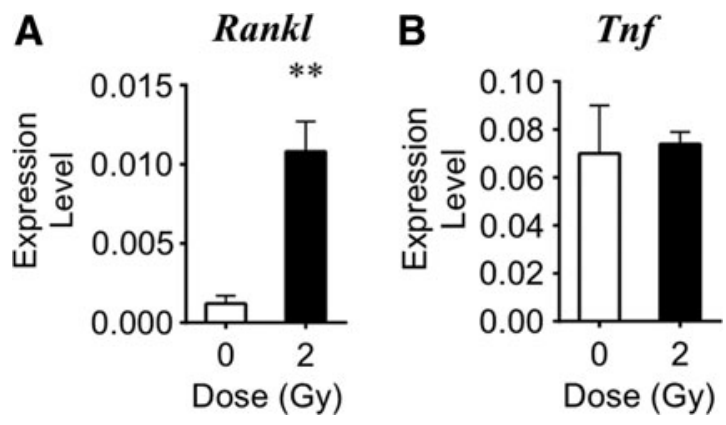

FIG. 2. $2 \mathrm{~Gy}$ iron irradiation effects on cytokine gene expression in tibial marrow cells on day 3 (expression level normalized to L19). Radiation exposure increased the gene expression levels of (A) Rankl. Gene expression levels of (B) $\operatorname{Tnf}$ were unchanged. Data are mean $\pm \mathrm{SD}$, with $* *$ denoting $P<0.01$ versus sham.

\section{Marrow gene expression following iron irradiation}

To determine if irradiation altered expression of various pro-osteoclastogenic genes, mRNA levels in bone marrow cell lysates were measured using quantitative RT-PCR 3 days after exposure to high-LET iron or sham irradiation. Within 3 days of exposure, iron irradiation increased expression of the Rankl gene by 9.2-fold compared with shamirradiated controls (Fig. 2). At this time point, transcripts of Opg and Mcpl were not detected in the marrow (data not shown). Radiation exposure did not alter Tnf expression (Fig. 2). In contrast, a lower dose of iron (5 cGy) did not elicit changes in gene expression (data not shown). These data demonstrated that high-LET particulate irradiation with 2 Gy elicited pro-osteoclastogenic cytokine expression in the bone marrow tissue.

\section{Gene expression of skeletal tissue following iron irradiation}

To determine if ionizing radiation altered expression of select genes related to osteoclastogenesis (Rankl, Opg) and osteoclast-mediated bone resorption (Ctk, Acp5) in cells within the mineralized compartment of skeletal tissue, RNA was purified from bone after removal of the marrow (leaving predominantly osteocytes). Within 3 days, iron irradiation increased expression of Rankl by 1.9 -fold, Acp 5 by 1.5 -fold, and $C t k$ by 2.1-fold over sham controls (Fig. 3). Expression levels of $O p g$ did not change. The ratio of Rankl/Opg ex- pression increased 2.8 -fold, which provides a relative index that, on balance, cytokine levels favored increased bone resorption.

\section{Marrow gene expression following gamma irradiation}

To determine whether irradiation altered osteoclastogenic and inflammation-related genes, mRNA expression was measured in bone marrow at $4 \mathrm{~h}$ or 1,3 , or 7 days after lowLET gamma irradiation and in sham-irradiated controls. Within 4 h, 2 Gy gamma irradiation elevated Rankl in bone marrow by 2.6-fold over the sham; gene expression of $\mathrm{Opg}$ was undetectable regardless of treatment (data not shown). Subsequently, the expression of many genes of interest measured in bone marrow (Rankl, Csfl, Nfatc1, Tnf, Mcpl, and Il6) transiently increased within 1 day and subsequently declined toward sham levels (Fig. 4). Expression of Tnf remained elevated through day 3 post-irradiation, while high Rankl expression persisted through day 7 post-irradiation.

At their peak, expression of pro-osteoclastogenic genes, Rankl and Csfl, and the osteoclast-related transcription factor, Nfatc1, increased by 4.1-fold, 4.2-fold, and 2.0-fold, respectively (Fig. 4A, D, E); the Rankl decoy receptor Opg showed a trend toward increased expression by 11.3 -fold $(P=0.053$ by ANOVA, Fig. 4B); pro-inflammatory genes Tnf, Mcpl, and Il6 increased by 1.7-fold, 11.9-fold, and 1.6fold, respectively (Fig. 4F, G, H), relative to controls. The ratio of Rankl/Opg increased by 1.8-fold at day 7 after irradiation (Fig. 4C). Additionally, radiation exposure showed a trend toward increased gene expression of $N f e 2 l 2$ by 1.6fold on day 1 compared with controls $(P=0.061$ for the ANOVA, Fig. 4I). Notably, levels of $N f e 2 l 2$ expression were strongly and positively correlated with the osteoclast-related genes Nfatcl $(P<0.05, r=0.89)$, Csfl $(P<0.05, r=0.63)$, $\operatorname{Tnf}(P<0.05, r=0.62)$, and Rankl $(P<0.05, r=0.56)$. These data show the temporal dependence of changes in cytokine gene expression in the marrow following radiation exposure.

\section{Gene expression of skeletal tissue following gamma irradiation}

Following gamma irradiation, mRNA levels in femoral mineralized tissue (sans marrow) were quantified at 3 and 7 days after irradiation or sham using qRT-PCR normalized to the housekeeping gene, L19, as shown in Fig. 5. Within 3 days, gamma radiation exposure increased the expression of Rankl (2.3-fold), Acp5 (2.2-fold), and Ctk (2.3-fold). Expression of
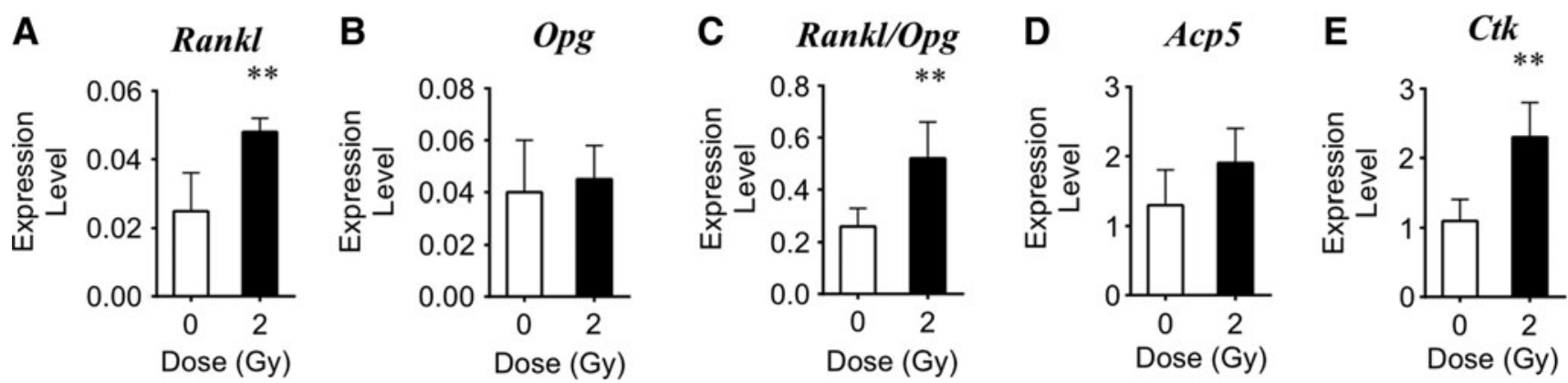

FIG. 3. 2 Gy iron irradiation effects on gene expression in tibial tissue (sans marrow) by day 3. Comparison of expression levels of (A) Rankl, (B) Opg, (C) Rankl/Opg, (D) Acp5, and (E) Ctk genes after iron irradiation compared with controls (expression level normalized to L19). Data are mean $\pm \mathrm{SD}$, with ** denoting $P<0.01$ versus sham. 
FIG. 4. 2 Gy gamma radiation increased expression of pro-osteoclastic and resorption-related genes in pooled tibial and femoral marrow (expression level normalized to L19). Time course $(+1,+3$, and +7 days post-irradiation) for the following genes compared with sham control: (A) Rankl, (B) Opg, (C) Rankll Opg, (D) Csf1, (E) Nfatc1, (F) Tnf, (G) Mcpl, (H) Il6, and (I) Nfe2l2. Data are mean $\pm \mathrm{SD}$, with $*$ denoting $P<0.05$ and $* * P<0.01$ versus sham and \# denoting $P=0.061$ for the ANOVA.
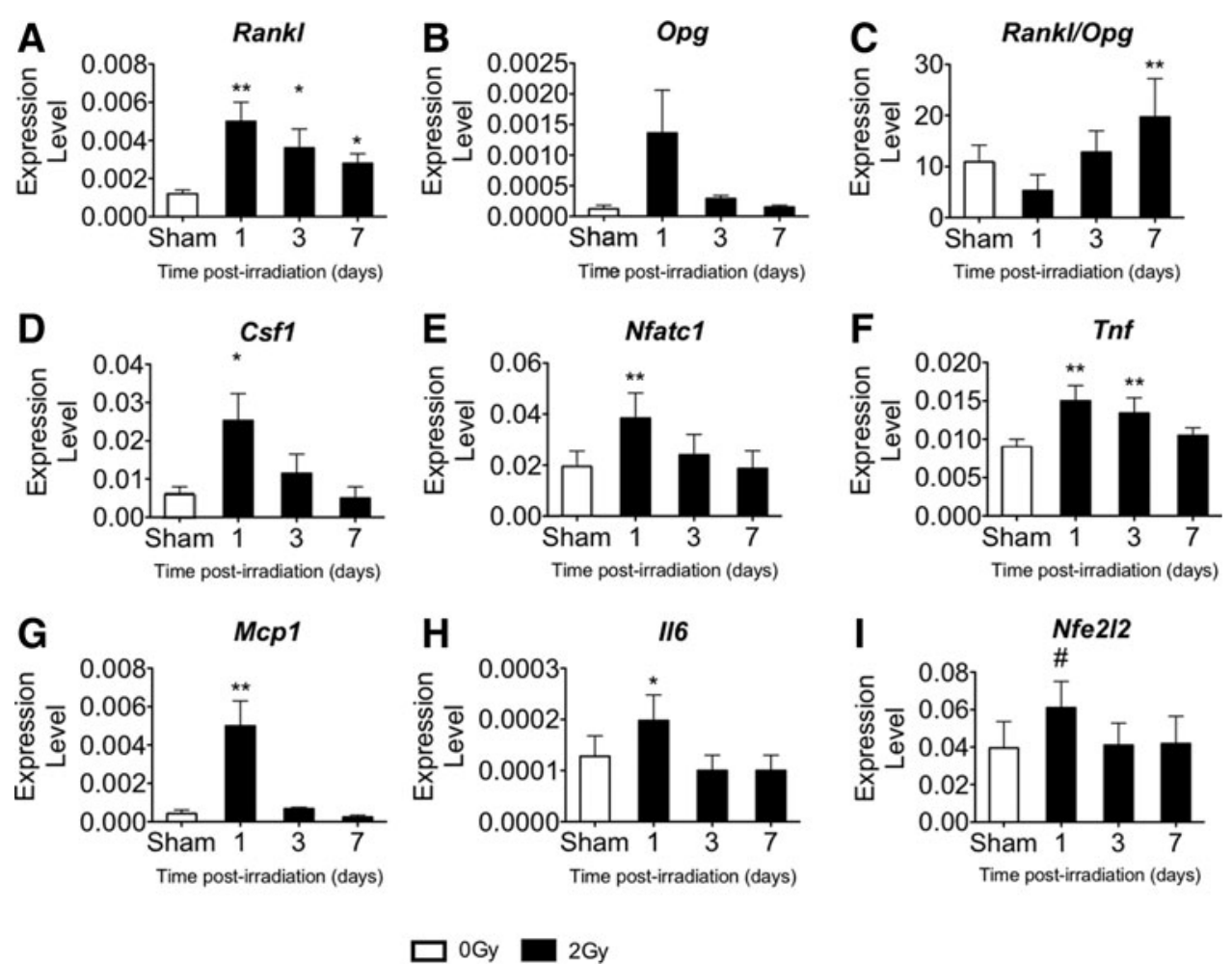

radiation increased TRACP $5 \mathrm{~b}$ serum levels by $34 \%$ on day 1 and $17 \%$ on day 3 compared with sham, with a subsequent gradual decline toward control levels by day 7 (data not shown). Thus, circulating TRACP 5b levels showed a similar time course, although lower in magnitude compared with skeletal gene expression for Rankl and other pro-osteoclastogenic cytokines.

\section{Discussion}

To better understand the mechanisms underlying radiationinduced stimulation of bone resorption, we investigated molecular signals within the latency period between
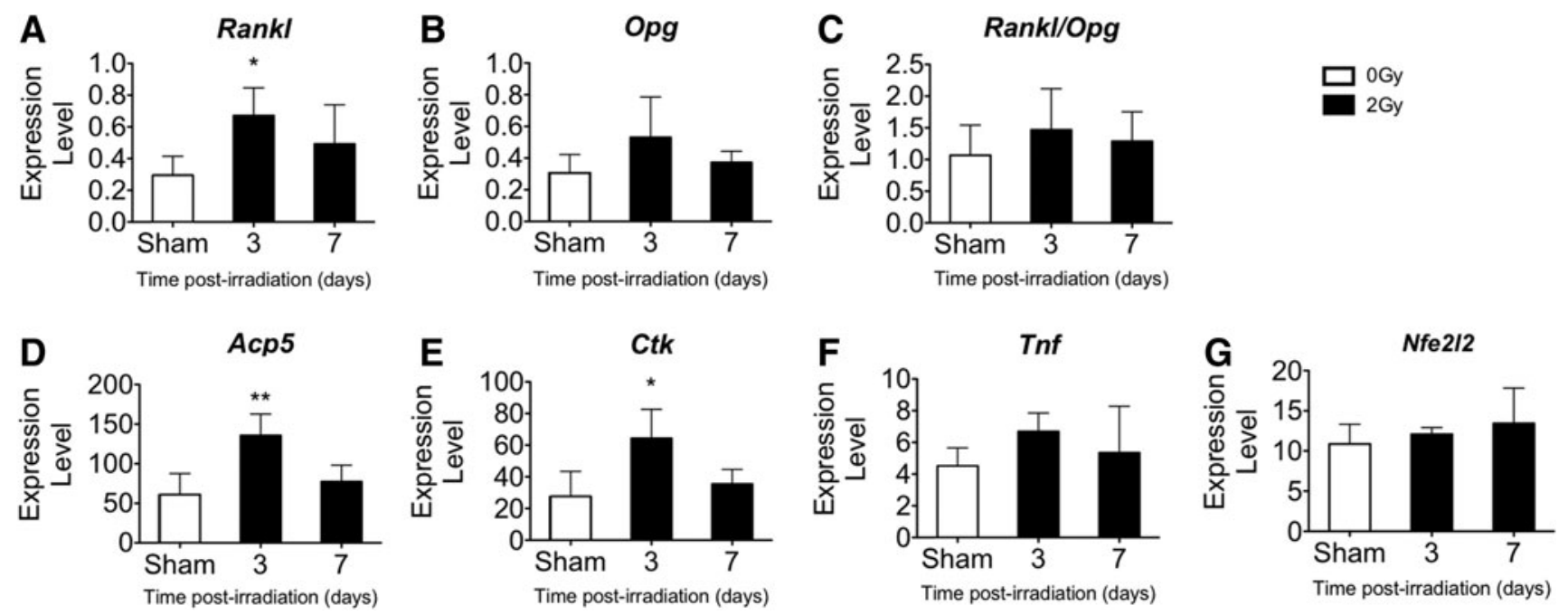

FIG. 5. 2 Gy gamma irradiation increased gene expression in femoral and tibial tissue (sans marrow) by day 3. Comparison of expression levels of (A) Rankl, (B) Opg, (C) Rankl/Opg, (D) Acp5, (E) Ctk, (F) Tnf, and (G) Nfe2l2 genes after iron irradiation compared with controls (expression level normalized to L19). Data are mean \pm SD, with $*$ denoting $P<0.05$ and ** denoting $P<0.01$ versus sham. 
radiation exposure and the manifestation of cancellous tissue loss. In summary, the results show that exposure to either gamma or heavy-ion radiation increased gene expression for the canonical osteoclastogenic factor, Rankl, as well as other pro-osteoclast cytokines (Mcpl, Tnf, Il6); this occurred in both marrow and mineralized tissue. These findings do not distinguish between changes in gene expression levels due to a shift in the cell population expressing osteoclastogenic cytokines, a generally higher level of expression for all the cells, or some combination of both. In fact, after exposure to radiation of sufficient dosage, there are pronounced, if transient, changes in marrow cell viability and increases in marrow macrophages, which are likely to contribute to the generation of new osteoclasts. Therefore, we interpret our results to show that exposure to ionizing radiation increased the tissue concentrations of mRNA levels generally so as to favor osteoclastogenesis.

Radiation-induced changes in cytokine expression were temporally related to changes in several indices of bone resorption, including gene expression (Ctk, Acp5, Nfatcl) and a serum biomarker (TRACP 5b levels). In addition, marrow expression of the global antioxidant transcription factor, $\mathrm{Nfe} 2 \mathrm{l} 2$, showed high correlation with the expression levels of several osteoclast-related genes. These data indicate that the activation of oxidative defenses and of osteoclast formation was temporally associated in response to radiation exposure. Our previous work showed radiation-induced oxidative damage presents later, at 10 days after exposure (Kondo and others 2010). Taken together, our results indicate that $N f e 2 l 2$-related antioxidant defense pathways were insufficient to prevent oxidative damage in bone.

Changes in gene expression (as early as $4 \mathrm{~h}$ or 1 day) preceded bone loss, which manifested in these experiments by day 7 after exposure to 2 Gy iron, although in some cases such as gamma irradiation and lower doses of iron, decrements in cancellous bone volume can be observed as early as 3 days after exposure (Kondo and others 2009; Yumoto and others 2010). Furthermore, a dose threshold was observed, as 5 cGy iron exposure failed to elicit changes in cytokine gene expression in the marrow or cancellous bone structure (data not shown); these findings provide additional indirect evidence in support of the hypothesis that early induction of osteoclastogenic cytokine gene expression by biologically effective doses of radiation leads to cancellous bone loss.

Together, the results demonstrate that radiation-induced structural changes were associated with a marrow environment favoring stimulation of osteoclast differentiation and activity by Rankl and other inflammation-related cytokines as follows: Radiation increased gene expression levels for proosteoclastogenic signaling molecules (Csfl, Rankl, Tnf), as well as a trend toward increasing antiosteoclastogenic molecules $(O p g)$, in the marrow and mineralized tissue of irradiated mice compared with sham controls. Iron irradiation elevated the ratio of Rankl/Opg in both the marrow and skeletal tissue by day 3, whereas after gamma irradiation, the ratio was elevated in the marrow at $4 \mathrm{~h}$ and then again at day 7 .

A rapid stimulation of Rankl expression by irradiation in the marrow and bone compartment, potentially from hematopoietic lineage cells (Pacifici 2010, 2012; Fumoto and others 2014) or stromal lineage cells (Suda and others 1999; Boyle and others 2003), is consistent with studies that used radiotherapeutic doses and regimens. In young mice, a sin- gle dose of 5 or $10 \mathrm{~Gy}$ increased Rankl/Opg in whole femora within 3 days (Han and others 2014). Using mice deficient in the antioxidant transcription factor, $N f e 2 l 2$, radiation exposure at high dose (20 Gy) increased RNA expression for Rankl in cultured osteoblasts grown ex vivo, but this was not observed in cells from wild-type mice (Rana and others 2012). These findings suggest an Nfe2l2-mediated increase in expression of antioxidant enzymes may dampen bone resorption responses to radiation.

Furthermore, when a macrophage cell line (RAW264.7) capable of differentiating into osteoclast-like cells after RANKL treatment is exposed to ionizing radiation (2 Gy gamma), gene expression levels rise for $\beta 3$ integrin, an adhesion receptor that is important for osteoclast differentiation, as well as receptor activator of nuclear factor kappaB (Rank), the receptor for Rankl on osteoclasts (Yang and others 2012). In other in vitro work, irradiation with 2 or 4 Gy increases Rankl in differentiated, MC3T3-E1 osteoblast-like cells (Yang and others 2013). Our work is the first to demonstrate the time course of radiation-induced changes in expression of various osteoclastogenic cytokines following 2 Gy exposure (both low- and high-LET) with subsequent bone loss and within the upper range of space-relevant doses and types of radiation.

Ionizing radiation also increased tissue expression of proinflammatory, osteoclastogenic ligands, Mcpl, Tnf, and Il6, which are all factors generally thought to stimulate osteoclast activity (Takayanagi 2007) in the presence of RANKL (Kostenuik and Shalhoub 2001; Yu and others 2004; Kim and others 2005, 2006; Sul and others 2012; Liu and others 2013). In some reports, TNF may act independently of RANKL to stimulate osteoclastogenesis (Kobayashi and others 2000). Our results are consistent with other work showing that in vivo exposure to ionizing radiation leads to rapid, complex, and interrelated sequence of signals constituting an immune-related cytokine response in bone marrow (Schaue and McBride 2010; Willey and others 2011; Schaue and others 2012; Buchwald and Aurora 2013). At a very high dose $(10 \mathrm{~Gy})$, which is sufficient to ablate the bone marrow of hematopoietic cells, radiation causes bone loss related to elevated fractalkine expression by vascular endothelial cells, inflammatory cytokines $T n f$, interleukin 1 beta, and interferon gamma, and recruitment of preosteoclasts (CD11b) (Han and others 2014). Additionally, the time course of cytokine and resorption-related gene expression shown in this study coincides with that of marrow cell death and repopulation (Otsuka and others 2008; Kondo and others 2010), suggesting a possible relationship between an expanded population of marrow macrophages clearing apoptotic cells and debris after irradiation, the differentiation of macrophages into osteoclasts, and increased resorption activity.

Taken together, these data lead us to propose a 2-stage process for radiation-induced osteoclastogenesis: first, radiation-induced gene expression of osteoclastogenic cytokines leads to enrichment of the marrow with osteoclast precursors (monocyte-macrophage, myeloid lineage cells) and drives differentiation into osteoclasts, and, second, that inflammation-related cytokines co-stimulate differentiating and mature osteoclasts.

As a functional measure of active osteoclasts, we provide evidence that $2 \mathrm{~Gy}$ gamma irradiation elevated circulating levels of osteoclast-specific TRACP 5b protein, indicative of 
increased bone resorption. In this work, the radiation-induced elevation in serum TRACP $5 b$ returned to control levels by day 7 following exposure, These results are consistent with those reported by Willey and others (2010, 2011) showing that $X$-irradiation ( $2 \mathrm{~Gy}$ ) of female mice increases circulating TRACP $5 b$ levels 1,3 , and 7 days after irradiation.

Radiation-induced decrements in cancellous tissue observed in these experiments were consistent with our previous results. Acute cancellous bone loss temporally manifests on day 3 at a dose as low as 10 cGy iron (Yumoto and others 2010). Persistent structural decrements (lasting $>1$ week) manifest at doses above $\sim 50 \mathrm{cGy}$ iron (Yumoto and others 2010) and 1 Gy gamma exposure (Hamilton and others 2006; Kondo and others 2010) before being overtaken, in the case of gamma irradiation, by age-related bone loss (Alwood and others 2012). Taken together, given the observed time course of skeletal gene expression and serum resorption markers, we conclude that the structural deficits arose from a spike in osteoclastogenic cytokine expression that follows exposure to ionizing radiation (Kondo and others 2009; Willey and others 2010).

Limitations of this work include the dose rate used to model space radiation. It is an open question whether lower dose rate exposures that constitute true space radiation stimulate osteoclasts and bone loss to the same extent as the exposures used here. In addition, other signaling molecules, including various other cytokines not studied here, are likely also to play a role in regulating bone resorption after challenge with ionizing radiation, while changes in osteoblast lineage cells are likely to be important for maintaining structural integrity at later times post-irradiation.

In conclusion, an improved understanding of the molecular response to radiation exposure may aid the development of biological treatments to mitigate potentially deleterious skeletal consequences during space flight.

\section{Acknowledgments}

This research was supported by the National Space Biomedical Research Institute grant No. MA02501 under NASA cooperative agreement NCC 9-58 (R.K.G., J.S.A.), a DOE-NASA Interagency Award No. DE-SC0001507, supported by the Office of Science (Biological and Environmental Research), U.S. Department of Energy (R.K.G.), and 2 NASA Postdoctoral Program fellowships from NASA's Space Biology Program (J.S.A., A.K). The authors thank P. Guida, A. Rusek, L. Loudenslager, and A. Kim of the NASA Space Radiation Laboratory at BNL for experimental support and S. Choi and T. Truong for microcomputed tomography support and C. Tahimic for manuscript review.

\section{Author Disclosure Statement}

No competing financial interests exist.

\section{References}

Alwood JS, Kumar A, Tran LH, Wang A, Limoli CL, Globus RK. 2012. Low-dose, ionizing radiation and age-related changes in skeletal microarchitecture. J Aging Res 2012:481983.

Alwood JS, Yumoto K, Mojarrab R, Limoli CL, Almeida EA, Searby ND, Globus RK. 2010. Heavy ion irradiation and un- loading effects on mouse lumbar vertebral microarchitecture, mechanical properties and tissue stresses. Bone 47:248-255.

Baxter NN, Habermann EB, Tepper JE, Durham SB, Virnig BA. 2005. Risk of pelvic fractures in older women following pelvic irradiation. JAMA 294(20):2587-2593.

Bouxsein ML, Boyd SK, Christiansen BA, Guldberg RE, Jepsen KJ, Muller R. 2010. Guidelines for assessment of bone microstructure in rodents using micro-computed tomography. $\mathrm{J}$ Bone Miner Res 25(7):1468-1486.

Boyce BF, Xing L. 2008. Functions of RANKL/RANK/OPG in bone modeling and remodeling. Arch Biochem Biophys 473(2):139-146.

Boyle WJ, Simonet WS, Lacey DL. 2003. Osteoclast differentiation and activation. Nature 423(6937):337-342.

Braun T, Schett G. 2012. Pathways for bone loss in inflammatory disease. Curr Osteoporos Rep 10(2):101-108.

Buchwald ZS, Aurora R. 2013. Osteoclasts and CD8 T cells form a negative feedback loop that contributes to homeostasis of both the skeletal and immune systems. Clin Dev Immunol 2013:429373.

Durante M, Cucinotta FA. 2011. Physical basis of radiation protection in space travel. Rev Mod Phys 83(4):1245-1281.

Fumoto T, Takeshita S, Ito M, Ikeda K. 2014. Physiological functions of osteoblast lineage and T cell-derived RANKL in bone homeostasis. J Bone Miner Res 29(4):830-842.

Hamilton SA, Pecaut MJ, Gridley DS, Travis ND, Bandstra ER, Willey JS, Nelson GA, Bateman TA. 2006. A murine model for bone loss from therapeutic and space-relevant sources of radiation. J Appl Physiol 101(3):789-793.

Han KH, Ryu JW, Lim KE, Lee SH, Kim Y, Hwang CS, Choi JY, Han KO. 2014. Vascular expression of the chemokine CX3CL1 promotes osteoclast recruitment and exacerbates bone resorption in an irradiated murine model. Bone 61:91101.

Hassler DM, Zeitlin C, Wimmer-Schweingruber RF, Ehresmann B, Rafkin S, Eigenbrode JL, Brinza DE, Weigle G, Bottcher S, Bohm E, Burmeister S, Guo J, Kohler J, Martin C, Reitz G, Cucinotta FA, Kim MH, Grinspoon D, Bullock MA, Posner A, Gomez-Elvira J, Vasavada A, Grotzinger JP. 2014. Mars' surface radiation environment measured with the Mars Science Laboratory's Curiosity rover. Science 343(6169):1244797.

Hyeon S, Lee H, Yang Y, Jeong W. 2013. Nrf2 deficiency induces oxidative stress and promotes RANKL-induced osteoclast differentiation. Free Radic Biol Med 65:789-799.

Keyak JH, Koyama AK, LeBlanc A, Lu Y, Lang TF. 2009. Reduction in proximal femoral strength due to long-duration spaceflight. Bone 44(3):449-453.

Kim MS, Day CJ, Morrison NA. 2005. MCP-1 is induced by receptor activator of nuclear factor- $\{$ kappa $\} \mathrm{B}$ ligand, promotes human osteoclast fusion, and rescues granulocyte macrophage colony-stimulating factor suppression of osteoclast formation. J Biol Chem 280(16):16163-16169.

Kim MS, Day CJ, Selinger CI, Magno CL, Stephens SR, Morrison NA. 2006. MCP-1-induced human osteoclast-like cells are tartrate-resistant acid phosphatase, NFATc1, and calcitonin receptor-positive but require receptor activator of NFkappaB ligand for bone resorption. J Biol Chem 281(2):1274-1285.

Kobayashi K, Takahashi N, Jimi E, Udagawa N, Takami M, Kotake S, Nakagawa N, Kinosaki M, Yamaguchi K, Shima N, Yasuda H, Morinaga T, Higashio K, Martin TJ, Suda T. 2000. Tumor necrosis factor alpha stimulates osteoclast differentiation by a mechanism independent of the ODF/ RANKL-RANK interaction. J Exp Med 191(2):275-286. 
Kobayashi M, Yamamoto M. 2005. Molecular mechanisms activating the Nrf2-Keap1 pathway of antioxidant gene regulation. Antioxid Redox Signal 7(3-4):385-394.

Kondo H, Searby ND, Mojarrab R, Phillips J, Alwood J, Yumoto K, Almeida EA, Limoli CL, Globus RK. 2009. Totalbody irradiation of postpubertal mice with (137)Cs acutely compromises the microarchitecture of cancellous bone and increases osteoclasts. Radiat Res 171(3):283-289.

Kondo H, Yumoto K, Alwood JS, Mojarrab R, Wang A, Almeida EA, Searby ND, Limoli CL, Globus RK. 2010. Oxidative stress and gamma radiation-induced cancellous bone loss with musculoskeletal disuse. J Appl Physiol 108:152-161.

Kostenuik PJ, Shalhoub V. 2001. Osteoprotegerin: a physiological and pharmacological inhibitor of bone resorption. Curr Pharm Des 7(8):613-635.

Lang T, LeBlanc A, Evans H, Lu Y, Genant H, Yu A. 2004. Cortical and trabecular bone mineral loss from the spine and hip in long-duration spaceflight. $\mathrm{J}$ Bone Miner Res 19(6):1006-1012.

Lang TF, Leblanc AD, Evans HJ, Lu Y. 2006. Adaptation of the proximal femur to skeletal reloading after long-duration spaceflight. J Bone Miner Res 21(8):1224-1230.

LeBlanc A, Schneider V, Shackelford L, West S, Oganov V, Bakulin A, Voronin L. 2000. Bone mineral and lean tissue loss after long duration space flight. J Musculoskelet Neuronal Interact 1(2):157-160.

Liu Z, Xu J, Li H, Zheng Y, He J, Liu H, Zhong Y, Lu Y, Hong B, Zhang M, Lin P, Du J, Hou J, Qian J, Kwak LW, Yi Q, Yang J. 2013. Bone marrow stromal cells derived MCP-1 reverses the inhibitory effects of multiple myeloma cells on osteoclastogenesis by upregulating the RANK expression. PLoS One 8(12):e82453.

Lloyd SA, Bandstra ER, Willey JS, Riffle SE, Tirado-Lee L, Nelson GA, Pecaut MJ, Bateman TA. 2012. Effect of proton irradiation followed by hindlimb unloading on bone in mature mice: a model of long-duration spaceflight. Bone 51(4):756-764.

Otsuka K, Koana T, Tomita M, Ogata H, Tauchi H. 2008. Rapid myeloid recovery as a possible mechanism of whole-body radioadaptive response. Radiat Res 170(3):307-315.

Pacifici R. 2010. T cells: critical bone regulators in health and disease. Bone 47(3):461-471.

Pacifici R. 2012. Role of T cells in ovariectomy induced bone loss—revisited. J Bone Miner Res 27(2):231-239.

Parsons JL, Townsend LW. 2000. Interplanetary crew dose rates for the August 1972 solar particle event. Radiat Res 153(6):729-733.

Rana T, Schultz MA, Freeman ML, Biswas S. 2012. Loss of Nrf2 accelerates ionizing radiation-induced bone loss by upregulating RANKL. Free Radic Biol Med 53(12):2298-2307.

Schaue D, Kachikwu EL, McBride WH. 2012. Cytokines in radiobiological responses: a review. Radiat Res 178(6):505-523.

Schaue D, McBride WH. 2010. Links between innate immunity and normal tissue radiobiology. Radiat Res 173(4):406-417.

Shurshakov VA, Petrov VM, Ivanov Yu V, Bondarenko VA, Tzetlin VV, Makhmutov VS, Dachev TsP, Semkova JV. 1999. Solar particle events observed on MIR station. Radiat Meas 30(3):317-325.
Suda T, Takahashi N, Udagawa N, Jimi E, Gillespie MT, Martin TJ. 1999. Modulation of osteoclast differentiation and function by the new members of the tumor necrosis factor receptor and ligand families. Endocr Rev 20(3):345-357.

Sul OJ, Ke K, Kim WK, Kim SH, Lee SC, Kim HJ, Kim SY, Suh JH, Choi HS. 2012. Absence of MCP-1 leads to elevated bone mass via impaired actin ring formation. J Cell Physiol 227(4):1619-1627.

Takayanagi H. 2007. Osteoimmunology: shared mechanisms and crosstalk between the immune and bone systems. Nat Rev Immunol 7(4):292-304.

Turner RT, Iwaniec UT, Wong CP, Lindenmaier LB, Wagner LA, Branscum AJ, Menn SA, Taylor J, Zhang Y, Wu H, Sibonga JD. 2013. Acute exposure to high dose gammaradiation results in transient activation of bone lining cells. Bone 57(1):164-173.

Willey JS, Livingston EW, Robbins ME, Bourland JD, TiradoLee L, Smith-Sielicki H, Bateman TA. 2010. Risedronate prevents early radiation-induced osteoporosis in mice at multiple skeletal locations. Bone 46(1):101-111.

Willey JS, Lloyd SA, Nelson GA, Bateman TA. 2011. Ionizing radiation and bone loss: space exploration and clinical therapy applications. Clin Rev Bone Miner Metab 9(1):54-62.

Yang B, Tang Q, Post J, Zhou H, Huang XB, Zhang XD, Wang Q, Sun YM, Fan FY. 2013. Effect of radiation on the Notch signaling pathway in osteoblasts. Int J Mol Med 31(3):698706.

Yang B, Zhou H, Zhang XD, Liu Z, Fan FY, Sun YM. 2012. Effect of radiation on the expression of osteoclast marker genes in RAW264.7 cells. Mol Med Rep 5(4):955-958.

Yu X, Huang Y, Collin-Osdoby P, Osdoby P. 2004. CCR1 chemokines promote the chemotactic recruitment, RANKL development, and motility of osteoclasts and are induced by inflammatory cytokines in osteoblasts. J Bone Miner Res 19(12):2065-2077.

Yumoto K, Globus RK, Mojarrab R, Arakaki J, Wang A, Searby ND, Almeida EA, Limoli CL. 2010. Short-term effects of whole-body exposure to (56)fe ions in combination with musculoskeletal disuse on bone cells. Radiat Res 173(4):494-504.

Zeitlin C, Hassler DM, Cucinotta FA, Ehresmann B, WimmerSchweingruber RF, Brinza DE, Kang S, Weigle G, Bottcher S, Bohm E, Burmeister S, Guo J, Kohler J, Martin C, Posner A, Rafkin S, Reitz G. 2013. Measurements of energetic particle radiation in transit to Mars on the Mars Science Laboratory. Science 340(6136):1080-1084.

Address correspondence to: Dr. Ruth K. Globus

Bone and Signaling Laboratory Space Biosciences Division NASA Ames Research Center Mail Stop 236-7

Moffett Field, CA 94035

E-mail: ruth.k.globus@nasa.gov

Received 2 September 2014/Accepted 31 December 2014 